\title{
Hybrid Variable-Scale Clustering Method for Social Media Marketing on User Generated Instant Music Video
}

\author{
Ai WANG, Xuedong GAO
}

\begin{abstract}
Social media has already become one of the mainstream enterprise marketing channels recently. That consists of various media elements, such as text, picture, and even newly developed instant music video etc. Although several text or picture mining techniques could be directly utilized to analyse online user comments, few researches focus on how to improve the marketing performance of social media platforms through a multimedia approach. Therefore, this paper studies the social media marketing problem of user generated instant music video. A hybrid variable-scale clustering algorithm (HVSC) is proposed to analyse user feature through both textual and video content. Combining with the information dissemination characteristics of social media platforms, we also put forth a marketing strategy that intensively enlarges the transmission audience of influential UGC videos. Experiment results show that the HVSC is able to support managers to discover the target potential customer base of each UGC video following their music preference and current interest/concerns. Finally, according to the content relevance and customer influence, the video producers' incentive mechanism is further discussed.
\end{abstract}

Keywords: scale transformation; social media mining; user-generated content; video marketing

\section{INTRODUCTION}

Social media has developed into one of the mainstream channels for enterprises marketing products or service recently, as over $90 \%$ of the firms and brands allocate specific personnel to operate their official sites on different social media platforms, such as Facebook, Twitter, and YouTube $[1,2]$. In terms of content producers, posts on social media can be classified as Firm Generated Content (FGC) and User Generated Content (UGC) that respectively reflects the social advertising (about a brand) and wisdom of crowds [3]. Although FGC is designed and examined by professional marketing team, studies find that brand-related UGC could achieve even better performance on plenty of marketing metrics due to the creativity and credibility $[4,5]$.

UGC on various social media platforms commonly consists of basic media elements, e.g., text, picture, audio and video [6]. According to the media richness theory [7], a video, that is a multimedia presentation, has great advantage on conveying the meaning of conditional events and facilitating understanding through the establishment of logical connections between symbolic systems, compared to text, picture and audio alone. Besides, advanced social media platforms even combine all these media elements together in order to enrich user experience, which is the birth of instant music videos (also called short videos) [8].

The latest statistical report on the development of Internet in China [9] verified that social media users of instant music videos, including Douyin, Kuaishou, Huoshan etc., accounted for $73.4 \%$ of the total mobile phone netizens in 2018, amounting to 578 million. Moreover, the research institution, Sensor Tower, showed that overseas version TikTok (Douyin in China) overtaken Facebook, Instagram, and YouTube have been awarded the most popular iOS application in APP Store with 45.8 million downloads till the first quarter of 2018 [10].

The short video marketing is an emerging research field on the basis of the traditional area, video marketing. On the one hand, video processing techniques gain a lot of attention. For example, Shi et al. [11] proposes an algorithm of object detection in short video based on the deep learning, which is successfully utilized in the content recommendation of product advertising. On the other hand, antecedent factors that influence user behaviours during video marketing also draw much awareness. Alamaki et al. [8] study the mobile video marketing problem of tourism service, and find that video design and online interaction are able to trigger users' participation intention and recalling scores. However, few researches focus on the marketing strategies and methods of user generated instant music video on social media in the perspective of firms and brands. That could not only advertise their products or service with the help of users' creativity and resources at a low cost, but also enlarge the interaction ways to potential customers.

Therefore, this paper studies the business marketing problem of user generated instant music video on social media. The main contributions are as follows. Firstly, we improve the scale transformation theory and propose the hybrid variable-scale clustering algorithm (HVSC) for feature recognition of social media users. Secondly, we apply our proposed method to the destination marketing of the Palace Museum using the open data source on Douyin. Experiment results show that the HVSC is able to support managers discover target user cluster (potential customers) of a valuable instant music video following users' interest and preference. Thirdly, according to the content relevance and influence of an instant music video, incentive mechanism of video producers is also discussed.

The remainder of the paper is organized as follows. In Section 2 we present the previous work related to our research, including user generated content marketing, instant music video analysis and scale transformation theory. In Section 3, we describe our proposed method, the hybrid variable-scale clustering (HVSC). In Section 4, we conduct our experiment via the instant music video platform Douyin. The implications for theory and practice are also discussed. Finally, the paper is concluded in Section 5.

\section{LITERATURE REVIEW \\ 2.1 User Generated Content Marketing}

Unlike professional routines and practices, user generated content (UGC) is usually presented as a perceptual and extemporaneous expression on social media, 
deriving from novel inspiration or extreme emotional experience [1]. As a special type of UGC, brand-related UGC attracts much more attention of various firms and brands, even customers, due to its rich content and potential commercial value.

Since brand-related UGC plays such a significant role in social media marketing, it is necessary to understand what affects users' participation willingness to share their personal opinions and experience on these open platforms. Herrero et al [12] study this problem based on the Unified Theory of Acceptance and Use of Technology 2 (UTAUT2), and find that there are three key factors that promote users' intentions to participate in social media, i.e., performance expectancy, hedonic motivation, and habit. Nevertheless, the three other wildly mentioned elements, i.e. facilitating conditions, social influence, and privacy concerns, do not show any influence on users' involvement.

There are a lot of research testing the relationship between brand-related UGC and customers' purchasing behaviour, and most results are positive. Ukpabi et al. [13] illustrate that all the source characteristics, content characteristics, user characteristics and response effect could determine the UGC utilization of potential customers. Besides, source characteristics (i.e., credibility, expertise, trustworthiness and homophily) and content characteristics (i.e., novelty, valence, aesthetics, argument quality and information quality) act together on user characteristics (i.e., consumer profile, involvement, enjoyment, experience and benefit). Taking the social media element picture as an example, Paul [4] emphasizes that user generated picture belongs to the primary image formed by subjective personal experience, which is considered to be impartial and highly credible. On the contrary, the secondary image, created under objective and deliberate design, aims to meet concerned commercial metrics.

Moreover, Scholz et al. [14] analyze the dynamic effects of UGC and FGC with regard to consumer purchase behaviour through many economic target variables. Empirical analysis shows that the hierarchical structure of UGC and FGC on social media websites has a strong influence on the marketing effect. Alamaki et al. [8] examine the customer behaviour response to user generated video through measuring the recall rate, satisfaction and behavioural intention etc. Real experiments demonstrate that service video could provide positive effective on customer behaviour, especially during the information search stage. They also address the importance of reaching the right audience rather than producing high quality video with casual content, under marketing purpose.

\subsection{Instant Music Video Dissemination}

Although previous studies have already verified the effectiveness of UGC on products and service marketing, firms and brands are still caught in a dilemma that large number of user generated contents are definitely beneficial to the popularity and influence of their business, but will simultaneously boost additional pressure to discover highly relevant and commercial potential content. However, advanced social media platforms themselves have the recommendation function in order to facilitate and control the information dissemination of online users, which could directly be utilized in UGC marketing.

Taking the instant music video platform Douyin as an example, there are more than one recommendation algorithms that contribute to its information dissemination [10]. For a newly registered user, social media platform tends to recommend online content on the basis of the general information filled by user, commonly containing gender, age, education, vocation, revenue and location etc. That enables the collaborative filtering algorithm to find similar background users, and share their browsing history $[15,16]$. As we all know, social media is an open and comprehensive information interaction channel, platforms are not willing to force any user to conduct official identity authority. Hence, the recommendation accuracy in this stage is relatively low because of the information loss and distortion.

After entering the active usage stage, online users express their concerns and interest through browsing, commenting, thumbing up or retweeting the appealing content, which could be used to build the interest graph of social network. The interest graph reflects social relationships in the perspective of common majors, hobbies and topics that obviously improves the recommendation accuracy on social media [17, 18]. However, this information dissemination pattern causes fatigue and boring feeling after keeping watching the same types of content continuously, which easily does harm to the sustainable development of social media platforms.

Douyin has its original information dissemination approach that is superposition recommendation based on the content flow pool [10]. Douyin innovatively enlarges the recommendation of a single video from one-off task to rounds of ones, and establishes a comprehensive evaluation system of video performance on each round with gradient difference [19]. After an instant music video is published, platform will start the first round recommendation according to its natural attributes and labels, such as region, topic, fans, etc. If the video achieves a qualified score during first round, platform will launch the second round recommendation in a wider range of users. Therefore, a novel and welcome video will be sustained transmitted over time. That explains the reason why one million plus Likes video is commonly seen on Douyin.

\subsection{Scale Transformation Analysis}

Affected by comprehensive factors (like political factors, product quality, service level, etc.), empirical research and practical experience reveal that the key point to marketing success lies in customer understanding [20, 21]. Hence, effective methods and techniques should be utilized to help enterprises respond to customers' dynamic and complicated demands during marketing decision making.

The variable-scale clustering (VSC) is an object segmentation method based on the scale transformation, which expands the single-scale analysis process of traditional clustering methods to multi-scale analysis [22]. The VSC aims to obtain both available and accessible clusters for practical application, through outputting intrasimilar clusters (groups) with their clear scale description [23]. 
Previous research shows that the VSC could solve the social media marketing problem accurately and effectively. Wang et al. [24] studies the multifunctional product marketing on social media through combining the VSC with sentiment analysis, which respectively is responsible for customer segmentation and customer satisfaction prediction. Experiment results illustrate that the VSC is able to recognize the feature (that is interest and concerns) of every divided customer base, which could support managers making customer-centered marketing strategies and campaigns. A public opinion sensitivity index (POSI) is also proposed to filter potential target customers according to product-related online reviews.

However, the current variable-scale clustering algorithm is unable to solve the marketing problem of user generated instant music video due to several reasons. The concept space (CS) is the basic hierarchy structure model for scale transformation, which consists of two parts, i.e., concept chain and value space (see Fig. 1). The CS requires the relationship between value on different hierarchy should follow the partial order relation between their leading concepts, i.e., $C H_{i} \preccurlyeq C H_{k} \rightarrow V_{i j} \nsucc V_{k j}\left(j \in N^{+}\right)$, which ignore the numerical relation of measurable data $[25$, 26].

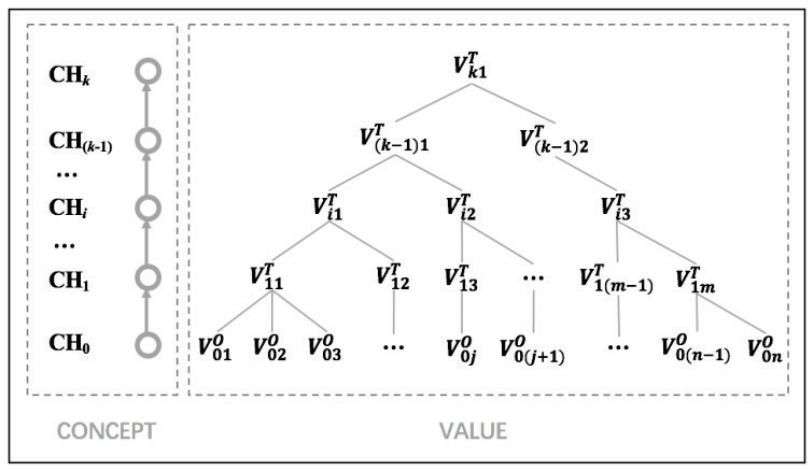

Figure 1 The concept space (CS)

After obtaining the initial dataset and CS, a multi-scale dataset could be built following business and data understanding [27-30]. There are two major functions for the multi-scale dataset: (1) strengthen the similarity of cluster feature during each iteration; (2) simplify the calculation complexity of meta clustering algorithm. The framework of the VSC is proposed based on the multi-scale dataset (see Fig. 2). Nevertheless, the VSC assumes that only one attribute could implement the scale transformation in each iteration. Although the scale transformation rate $(S T R)$ is able to optimize the attribute selection, the hypothesis still limits the improvement of algorithm efficiency. In addition, the multiple scale transformation demand could not be handled by the current framework.

Therefore, we improve the original variable-scale clustering algorithm for the instant music video marketing in the following Section 3.

\section{RESEARCH METHODS}

According to the media richness theory [7], an instant music video is the organic combination of three media elements, i.e., text, picture, and music (audio). Text and picture both present social media users' current concerns and new interest, while music selection reflects their aesthetic preference. Since there is a quantity constraint only on music selection of a user generated instant music video (i.e., no more than one song), it can be seen that the user producer stays in a one-to-one relation with music entity, but a one-to-many relation to text and pictures. Therefore, the previous single scale transformation methodology should be improved.

Definition 1: Given a dataset $D=D^{O}=$ $\left(U, A^{O}, V^{O}, f^{O}\right)$, its hybrid multi-scale dataset $D^{H S}=$ $\left(U, A^{T}, V^{T}, f^{T}\right)$, where $U$ is the universe of both $D^{O}$ and $D^{H S}, A^{O}$ is the original attribute set (original scale) of $D$, let $R \subseteq A^{O}$, if $|R|=1, A^{T}=\{C H(a), a \in R\}$ is the target attribute set (target scale) of $D, C H(a)$ is any concept in the CS of attribute $a$, and $A^{O} \preccurlyeq A^{T}, V^{O} \nsucc V^{T}$; if $|R|>1$, $A^{T}=\{\min (C H(a)), a \in R\}$ is the target attribute set (target scale) of $D, C H(a)$ is any value in the CS containing attribute $a$, and $A^{O} \preccurlyeq A^{T}, V^{O} \nsupseteq V^{T}$.

As for a hybrid multi-scale dataset (see Def. 1), the scale transformation of a single attribute could be accomplished following the partial order relation between concepts, while the scale transformation of multiple attributes could be achieved via the numerical relation between value situated in different hierarchy level.

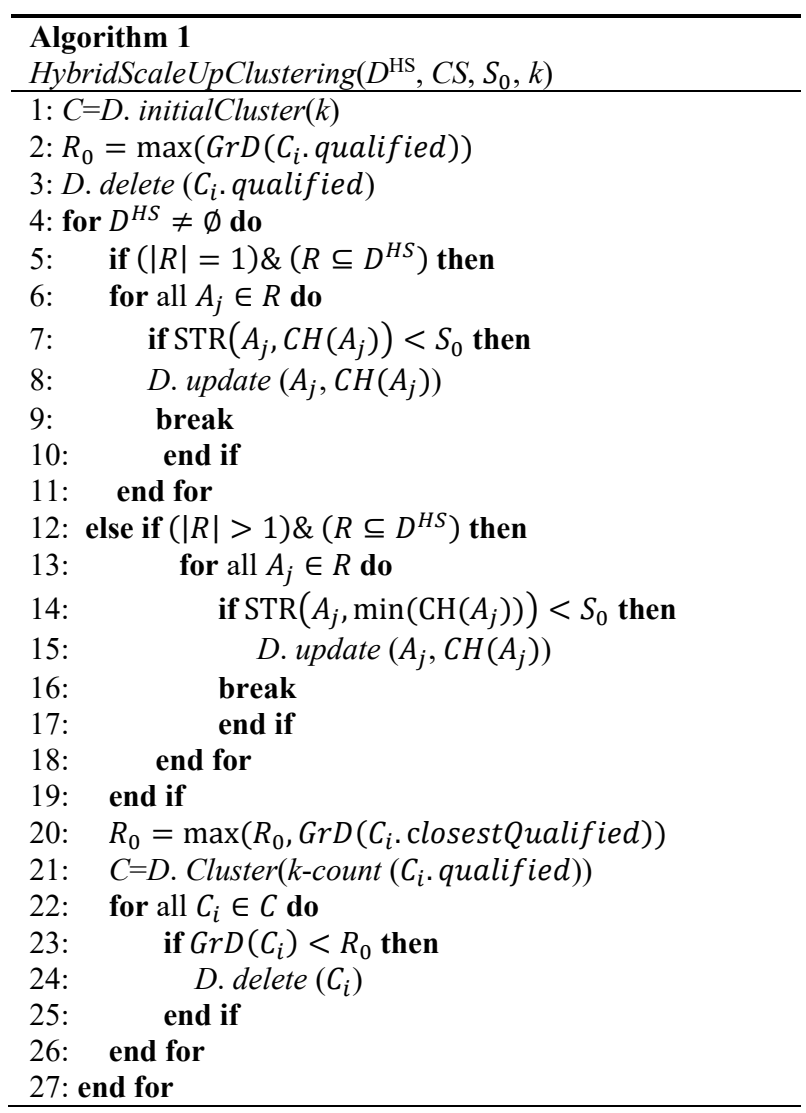

In order to control the attribute selection of scale transformation, the measurement, scale transformation rate $(S T R)$ is proposed in [22]

$\operatorname{STR}\left(A^{O}, A^{T}\right)=\sum_{i=1}^{n}\left|A^{T}{ }_{-}\left(A_{i}^{O}\right)\right| /|U|$

$A^{T}{ }_{-}\left(A_{i}^{O}\right)=\cup\left\{A_{j}^{T} \mid A_{j}^{T} \subseteq A_{i}^{O}\right\}$

Where 


$$
U / A^{O}=\left\{A_{1}^{O}, A_{2}^{O}, \ldots, A_{n}^{O}\right\}, \text { and } U / A^{T}=\left\{A_{1}^{T}, A_{2}^{T}, \ldots, A_{m}^{T}\right\}
$$

As for the single-scale transformation, $\operatorname{STR}\left(A^{O}, A^{T}\right)=$ $\operatorname{STR}(a, C H(\mathrm{a}))$, where $a \in R \subseteq A^{O}$ and $|R|=1$. As for the multiple-scale transformation, $\operatorname{STR}\left(A^{O}, A^{T}\right)=$ $\operatorname{STR}(R, C H(\mathrm{a}))$, where $a \in R \subseteq A^{O},|R|>1$, and $U / R=$ $\cap\{U / a\}$.

In terms of (marketing) decision making, the purpose of clustering method is to get all satisfying clusters instead of local optimal results, an evaluation metric to measure the discrete degree of each cluster is needed for the variable-scale clustering [22].

$$
\begin{aligned}
& \operatorname{Gr} D\left(C_{I}, A^{T}\right)=\sqrt{\sum_{j=1}^{m} \bar{d}\left(x_{i j}, x_{I j}\right) / G\left(U / A^{T}\right)} \\
& \bar{d}\left(x_{i j}, x_{I j}\right)=\sum_{i=1}^{n} \delta\left(x_{i j}, x_{I j}\right) / n
\end{aligned}
$$

$$
\begin{aligned}
& G\left(U / A^{T}\right)=\sum_{l=1}^{k}\left|A_{l}^{T}\right|^{2} / n^{2} \\
& \delta\left(x_{i j}, x_{I j}\right)=\left\{\begin{array}{l}
0, x_{i j},=x_{I j} \\
1, x_{i j}, \neq x_{I j}
\end{array}\right.
\end{aligned}
$$

where $x_{I j}$ is the mode of cluster $C_{I}, \bar{d}\left(x_{i j}, x_{I j}\right)$ is the mode deviation towards $x_{I j}$.

Similarly, the granular deviation $(G r D)$ of single-scale transformation is $\operatorname{GrD}\left(C_{I}, C H(a)\right)$, where $a \in R \subseteq A^{O}$ and $|R|=1$, and the granular deviation $(G r D)$ of multiplescale transformation is $\operatorname{GrD}\left(C_{I}, \min (C H(a))\right)$, where $a \in$ $R \subseteq A^{O}$ and $|R|>1$.

Finally, we propose the hybrid variable-scale clustering algorithm (HVSC), and the pseudocode is shown in Algorithm 1.

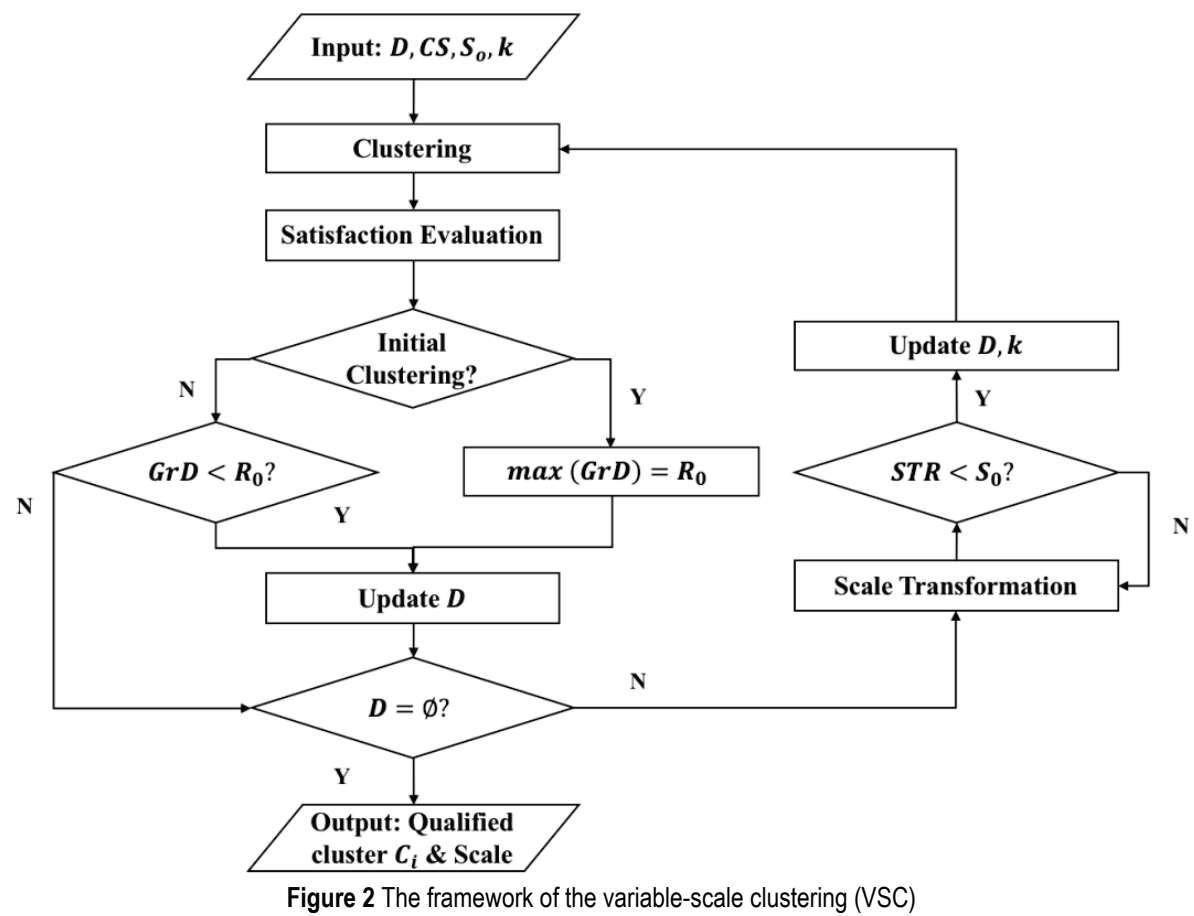

\section{RESULTS AND DISCUSSIONS}

China experiences a rapid growth in the number of network video users, especially short video users, in recent years [31, 32] (see Fig. 3). There were nearly 578 million instant music video users on social media in Jun 2018, up from 549 million six months before. Besides, the video software usage rate also stays in a relatively high level, amounting to $74 \%$ in average. Therefore, this paper selects the instant music video on social media as data source to implement our experiment.

Tab. 1 describes the data gathering process on the most popular iOS application Douyin (TikTok) [10]. We take an official account (called the cultural and creative pavilion of the Palace Museum) as an example, which has already passed the identity authentication. There are altogether four retrieval conditions: (1) User identity restriction. Only users that have already followed the official account are eligible because they are more likely to become customers in reality. Till Feb 8, 2019, there were over 30 thousand followers (fans) of that account in total, and we randomly select 1,000 of them as experimental samples. (2) User influence restriction. At least one of the three user influence measurements (the number of Likes, Fans, Works) must exceed the minimum level (as respectively $150,80,50)$. There are 144 instances of our sample set that satisfy the first two retrieval conditions, and become qualified users. (3) and (4) Video importance restrictions. Only the user generated videos or the user thumb-up videos with over one million Likes are chosen as our data acquisition objects.

We analyze historical usage records of all qualified users, and complete the data gathering following three dimensions, i.e., user, music, text \& picture. All the information could be directly obtained from Douyin client, except for four additional music attributes (shown in italics). These supplementary data are not only publicly available on the Internet and easy to access, but also beneficial for discovering user characteristic. Moreover, word segmentation is also conducted in order to better recognize user feature by comments and topic labels. 


\subsection{Experimental Results Analysis}

In this section, we implement user segmentation for instant music video marketing based on the HVSC, and results are shown in Tab. 2. It can be seen that all qualified users are divided into nine satisfy customer base (clusters), whose granular deviation is all under the maximum limit
$(G r D \leq 3.0682)$. Besides, each cluster has at least one influence metric (e.g., the average number of Likes, Fans, Works) that exceeds the minimum level, which further verifies the effectiveness of current customer segmentation results.

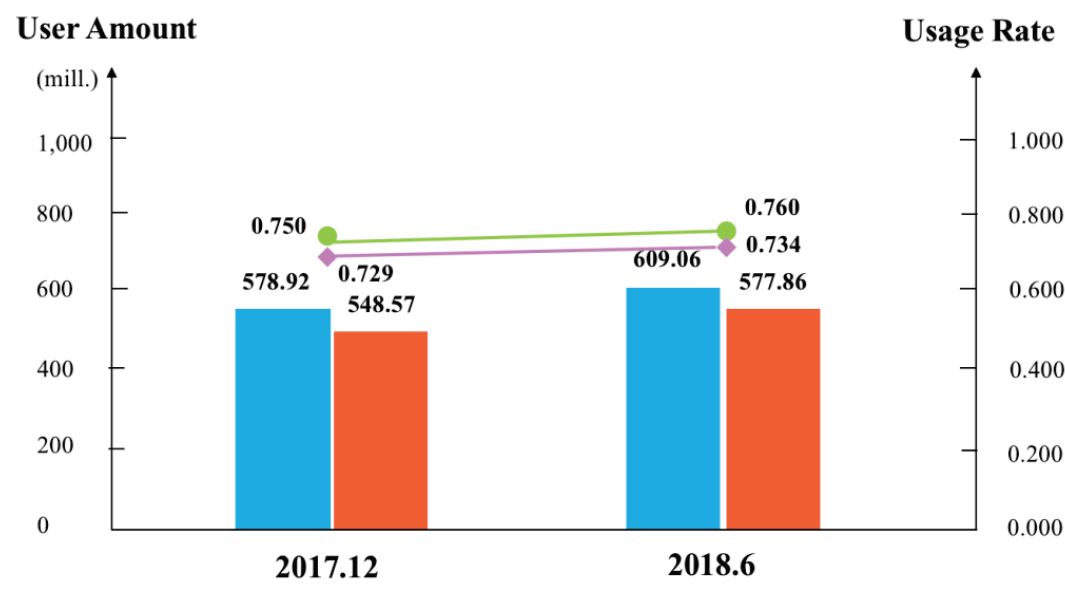

Figure 3 The user amount and usage rate of video platforms in China from Dec 2017 to Jun 2018 [9]

Table 1 The data gathering scheme

\begin{tabular}{|c|c|c|c|c|}
\hline Social Media & \multicolumn{4}{|c|}{ Douyin } \\
\hline Research Account & \multicolumn{4}{|c|}{ The cultural and creative pavilion of the Palace Museum (in Chinese) } \\
\hline Retrieval Conditions & \multicolumn{4}{|c|}{$\begin{array}{l}\text { (1) User that has followed the research account; } \\
\text { (2) User with minimum number of Likes (150), Fans (80), or Works (50); } \\
\text { (3) Qualified users' generated original instant music videos; } \\
\text { (4) Qualified users' thumb-up instant music videos with over one million Likes; }\end{array}$} \\
\hline & Object & User Information & Music Information & Text and Picture Information \\
\hline Data Structure & Data & $\begin{array}{l}\text { User id, } \\
\text { Number of Likes, } \\
\text { Number of Fans, } \\
\text { Number of Works }\end{array}$ & $\begin{array}{l}\text { Song name, Singer name, Singer gender, } \\
\text { First released time, Language, Music style }\end{array}$ & $\begin{array}{l}\text { Topic labels, User generated textual } \\
\text { comments, Topic labels of thumbed up } \\
\text { videos }\end{array}$ \\
\hline
\end{tabular}

\begin{tabular}{|c|c|c|c|c|c|c|c|c|}
\hline \multirow{2}{*}{ No. } & Cluster & $\begin{array}{c}\text { User } \\
\text { Count }\end{array}$ & $\begin{array}{c}\text { Avg. } \\
\text { Likes }\end{array}$ & $\begin{array}{c}\text { Avg. } \\
\text { Fans }\end{array}$ & $\begin{array}{c}\text { Avg. } \\
\text { Works }\end{array}$ & GrD & Music Preference & Interests / Concerns \\
\hline \multirow{2}{*}{1} & 1 & 22 & 106 & 183 & 15 & 2.8770 & A million possibilities (in Chinese) & New year, love, respect, security \\
\cline { 2 - 10 } & 2 & 26 & 831 & 199 & 24 & 3.0682 & Crying over you, Try TryTry & New year, dance, cat \\
\hline 2 & 3 & 10 & 80 & 306 & 48 & 2.7653 & Can I have WeChat (in Chinese) & Baby, love, celebrity \\
\hline 3 & 4 & 18 & 647 & 117 & 31 & 3.0458 & 2000 s, Pop music & Baby, house work, celebration, Han Chinese clothing \\
\hline \multirow{2}{*}{4} & 5 & 14 & 153 & 135 & 39 & $2.9478)$ & 2000s, Pop music, Chinese & Xi'an, celebrity, travel, Xi Jinpin \\
\cline { 2 - 10 } & 6 & 18 & 617 & 275 & 48 & 2.4401 & Travel, security, dog, baby & Love, cat, movie, Han Chinese clothing, \\
\hline \multirow{3}{*}{5} & 7 & 32 & 303 & 191 & 41 & 3.0255 & Chinese, Female singer & Video effects, New year, Hong Kong, lucky dog \\
\cline { 2 - 9 } & 8 & 2 & 179 & 45 & 28 & 1.5811 & Chinese, Male singer, Light music & Baby, house work, Alipay, New year \\
\cline { 2 - 9 }
\end{tabular}

In terms of customer feature, Clusters 1-3 have very clear music preference, that is a certain music song, which could directly guide the recommendation of user generated videos, which take the same song as background music. Generally, New Year, love and baby are three major concerns (interest) welcomed by most potential customers. Therefore, a business-related user generated video with these topic elements will win a larger audience. More than that, each customer base also has their own unique interest, such as Han Chinese clothing, celebrity, Hong Kong, etc., which could greatly help managers discover target customers and make marketing strategies.

\subsection{Incentive Mechanism of Target Customers}

There are two types of target customers for firms and brands during marketing process: (1) Users that generate business-related instant music videos. Since these users expect their original work to become popular and wildly be seen, incentive strategies should focus on expanding the transmission audience of their videos. (2) Users that have followed the business official account. As most of these users are fond of the products or service enterprise offered, increasing interaction and termly recommended newly attractive content would stimulate their interest. Hence, incentive mechanism in detail is proposed as follows.

Firstly, evaluate user generated instant music video according to its content relevance and customer influence. A video that covers more customer feature (i.e., music preference, as well as current interest/concerns) of target groups with large user count, wins a higher score.

Secondly, recommend high evaluation score videos to pre-segmented target customer base with the same interest/concerns, and gather their response and feedback. 
Additional online welfare could be provided to reward those users who accept business recommendation and disseminate it to their own followers (fans).

\section{CONCLUSIONS}

Video marketing on social media has become an emerging research field recently, and previous studies achieve great progress on the video processing techniques and video-driven influence factors of customer purchase behaviour. However, few researches focus on the marketing strategies and methods of user generated instant music video on social media in the perspective of firms and brands.

This paper studies the business marketing problem of user generated instant music video on social media. Firstly, we improve the scale transformation theory through expanding the single-scale transformation to multiple-scale transformation. A hybrid variable-scale clustering algorithm (HVSC) is also proposed for feature recognition of social media users. Secondly, we apply our proposed method to destination marketing of the Palace Museum using the open data source on Douyin. Experiment results show that the HVSC is able to support managers discover target user cluster (potential customers) of a valuable instant music video following users' music preference and current interest/concerns. Finally, firms and brands are able to promote marketing performance by establishing customer incentive mechanism, referring to the content relevance and customer influence gained by the HVSC.

The future research will focus on the improvement of HVSC algorithm efficiency by reducing iteration times for practical application.

\section{Acknowledgements}

The study is supported by national natural science foundation of China (71272161) and China Scholarship Council.

\section{REFERENCES}

[1] Roma, P. \& Aloini, D. (2019). How does brand-related usergenerated content differ across social media? Evidence reloaded. Journal of Business Research, 96(1), 322-339. https://doi.org/10.1016/j.jbusres.2018.11.055

[2] Jeong, B., Yoon, J., \& Lee, J. (2017). Social media mining for product planning: A product opportunity mining approach based on topic modeling and sentiment analysis. International Journal of Information Management. https://doi.org/10.1016/j.jijinfomgt.2017.09.009

[3] Colicev, A., Kumar, A., \& O'Connor, P. (2018). Modeling the relationship between firm and user generated content and the stages of the marketing funnel. International Journal of Research in Marketing, 36(1), 100-116. https://doi.org/10.1016/j.jiresmar.2018.09.005

[4] Paul, D. (2018). Characterizing the location of tourist images in cities. Differences in user-generated images (Instagram), official tourist brochures and travel guides. Annals of Tourism Research, 73(1), 103-115. https://doi.org/10.1016/j.annals.2018.09.001

[5] Hernandez, J., Kirilenko, A., \& Stepchenkova, S. (2018). Network approach to tourist segmentation via user generated content. Annals of Tourism Research, 73(1), 35-47. https://doi.org/10.1016/j.annals.2018.09.002
[6] Lim, H., Choi, J., Akhmedov, A., \& Chung, J. (2018). Predicting future trends of media elements in hotel marketing by using change propensity analysis. International Journal of Hospitality Management. https://doi.org/10.1016/j.ijhm.2018.10.001

[7] Lim, K. H. \& Benbasat, I. (2000). The effect of multimedia on perceived equivocality and perceived usefulness of information systems. MIS Quarterly, 24(3), 449-471. https://doi.org/10.2307/3250969

[8] Alamaki, A., Pesonen, J., \& Dirin, A. (2019). Triggering effects of mobile video marketing in nature tourism: Media richness perspective. Information Processing and Management, 56(3), 756-770. https://doi.org/10.1016/j.ipm.2019.01.003

[9] CNNIC. (2018). The $42^{\text {nd }}$ statistical report on the development of Internet in China. Beijing, 39-41. http://www.cac.gov.cn/2018-08/20/c_1123296882.htm

[10] Wang, H. (2018). Characteristic analysis of recommendation algorithms on Douyin. New Media Research, 20(1), 21-23. https://doi.org/10.16604/j.cnki.issn2096-0360.2018.20.007

[11] Shi, Y., Liu, S., \& Ma, C. (2018). Research on object detection and content recommendation system in short video based on deep learning. Computer and Modernization, 11(1), 69-76. https://doi.org/10.3969/j.issn.1006-2475.2018.11.014

[12] Herrero, A., Martin, H., \& Salmones, M. (2017). Explaining the adoption of social networks sites for sharing usergenerated content: a revision of the UTAUT2. Computer in Human Behavior, 71(1), 209-217. https://doi.org/10.1016/j.chb.2017.02.007

[13] Ukpabi, D. \& Karjaluoto, H. (2018). What drives travelers' adoption of user-generated content? A literature review. Tourism Management Perspectives, 28(1), 251-273. https://doi.org/10.1016/j.tmp.2018.03.006

[14] Scholz, M., Schnurbus, J., Haupt, H., Dorner, V., Landherr, A., \& Probst, F. (2018). Dynamic effects of user- and marketer- generated content on consumer purchase behavior: modeling the hierarchical structure of social media websites. Decision Support Systems, 113(1), 43-55. https://doi.org/10.1016/j.dss.2018.07.001

[15] Jeong, B., Yoon, J., \& Lee, J. (2017). Social media mining for product planning: A product opportunity mining approach based on topic modeling and sentiment analysis. International Journal of Information Management. https://doi.org/10.1016/j.ijinfomgt.2017.09.009

[16] Zhao, X., Zhan, M., \& Liu, B. F. (2018). Disentangling social media influence in crises: Testing a four-factor T model of social media influence with large data. Public Relations Review, 44(4), 549-561. https://doi.org/10.1016/j.pubrev.2018.08.002

[17] Shen, C., Chenb, M., \& Wang, C. (2018). Analyzing the trend of $\mathrm{O} 2 \mathrm{O}$ commerce by bilingual text mining on social media. Computers in Human Behavior. https://doi.org/10.1016/j.chb.2018.09.031

[18] Injadat, M., Salo, F., \& Nassif, A. B. (2016). Data mining techniques in social media: A survey. Neurocomputing, 214(1), 654-670. https://doi.org/10.1016/j.neucom.2016.06.045

[19] Qian, Y. (2018). Study on viral transmission from the perspective of usage and satisfaction: a case study of Douyin. Technology and Economic Guide, 26(36), 194-195.

[20] Guo, Y., Barnes, S. J., \& Jia, Q. (2017). Mining meaning from online ratings and reviews: Tourist satisfaction analysis using latent dirichlet allocation. Tourism Management, 59(1), 467-483. https://doi.org/10.1016/j.tourman.2016.09.009

[21] Geetha, M., Singha, P., \& Sinha, S. (2017). Relationship between customer sentiment and online customer ratings for hotels- An empirical analysis. Tourism Management, 61(1), 43-54. https://doi.org/10.1016/j.tourman.2016.12.022

[22] Gao, X. \& Wang, A. (2018). Variable-scale clustering. 2017 Int. Conf. Logist. Informatics Serv (LISS' 2018). In press. https://doi.org/10.1109/LISS.2018.8593262 
[23] Zhang, Q., Wang, G., \& Hu, J. (2013). Multi-granularity knowledge acquisition and uncertainty measurement. Beijing: Science Press, 1(1), 16-25.

[24] Wang, A. \& Gao, X. (2019). Multifunctional product marketing using social media based on the variable-scale clustering. Tehnicki vjesnik, 26(1), 193-200. https://doi.org/10.17559/TV-20181120082714

[25] Guo, Y., Barnes, S. J., \& Jia, Q. (2017). Mining meaning from online ratings and reviews: Tourist satisfaction analysis using latent dirichlet allocation. Tourism Management, 59(1), 467-483. https://doi.org/10.1016/j.tourman.2016.09.009

[26] Du, N. (2018). Study on short text data mining based on social media. Tianjin: Tianjin University of Technology.

[27] Wu, S., Gao, X., \& Bastien, M. (2003). Data warehousing and data mining. China: Metallurgical Industry Press, 9(1), 148-155.

[28] Sun, D. \& Zhao, S. (2015). Multi-scale clustering algorithm based on the weight vector, Computer Science, 42(1), 263 267.

[29] Han, Y. \& Zhao, S. (2016). Multi-scale clustering algorithm, Computer Science, 43(1), 244-248.

[30] Zhang, L. \& Zhang, B. (2003). Fuzzy quotient space theory, Journal of Software, 14(4), 770-776.

[31] Zhang, D., Sui, J., Gong, Y. (2017). Large scale software test data generation based on collective constraint and weighted combination method. Tehnicki vjesnik, 24(4), 1041-1050. https://doi.org/10.17559/TV-20170319045945

[32] Du, J. B., Sun, Y. L., Ren, H. Y. (2018). The Relationship of Delivery Frequency with the Cost and Resource Operational Efficiency: A Case Study of Jingdong Logistics. Mathematics and Computer Science, 3(6), 129-140. https://doi.org/10.11648/j.mcs.20180306.12

\section{Contact information:}

Ai WANG, PhD student (Corresponding author)

University of Science and Technology Beijing

No. 30 Xueyuan Road, Haidian District, 100083 Beijing, China

wangai22222@126.com

Xuedong GAO, Professor

University of Science and Technology Beijing,

No. 30 Xueyuan Road, Haidian District, 100083 Beijing, China

gaoxuedong@manage.ustb.edu.cn 